\title{
Gastrointestinal Manifestations in Hereditary Transthyretin Amyloidosis associated with Glu89Gln Mutation
}

\author{
Radislav Nakov ${ }^{1}$, Stayko Sarafov ${ }^{2}$, Ventsislav Nakov ${ }^{1}$, Mariana Gospodinova $^{3}$, Tihomir Todorov ${ }^{4}$, Andrey Kirov $^{4,5}$, \\ Albena Todorova $^{4,5}$, Ivailo Tournev ${ }^{2,6}$
}

1) Clinic of Gastroenterology,

Tsaritsa Joanna University

Hospital, Medical University

Sofia

2) Clinic of Nervous Diseases,

Alexandrovska University

Hospital, Medical University

Sofia

3) Clinic of Cardiology,

Medical Institute of Ministry

of Interior, Sofia

4) Genetic and Medico-

Diagnostic Laboratory

"Genica", Sofia

5) Department of Medical

Chemistry and Biochemistry,

Medical University, Sofia

6) Department of Cognitive

Science and Psychology, New

Bulgarian University,

Sofia, Bulgaria

Address for correspondence:

Radislav Nakov

8 Byalo more Str., Clinic of

Gastroenterology "Tsaritsa

Yoanna" University Hospital,

1527 Sofia, Bulgaria

radislav.nakov@gmail.com

Received: 17.08.2019

Accepted: 20.10.2019

\section{ABSTRACT}

Aims: In the current study we aimed to explore the prevalence of gastrointestinal (GI) manifestations in hereditary transthyretin amyloid (hATTR) amyloidosis associated with Glu89Gln mutation.

Methods: We recruited 78 patients with hATTR amyloidosis associated with Glu89Gln mutation. The diagnosis of hATTR was defined by a documented transthyretin mutation through DNA analysis. Symptoms were recorded as present or absent at the time of enrollment into the study. The gastrointestinal (GI) symptoms checklist included the following items: early satiety, nausea, vomiting, constipation, alternating diarrhea/ constipation, diarrhea, fecal incontinence and unintentional weight loss.

Results: Forty-two patients (53.8\%) reported at least one GI symptom or sign. Diarrhea was the most frequently reported (30.8\%), followed by unintentional weight loss (28.2\%) and nausea (21.8\%). Fecal incontinence (3.8\%) was the least common one. No significant gender related difference in overall GI symptom prevalence was found (females $52.16 \%$, males 55\%, p = 0.834). Type of disease onset was not related to GI prevalence (earlyonset $50 \%$, late-onset $55.6 \%, \mathrm{p}=0.650)$. After dividing the patients into groups with a disease duration of $<5$ years, 5-10 years and >10 years, respectively, the prevalence of GI symptoms was found to be significantly higher in later stages (26.3\% vs. $55.0 \%$ vs. $78.9 \%, \mathrm{p}=0.005$; OR 2.450, 95\% CI 1.084-5.538). Gastrointestinal manifestations had no impact on survival $(\mathrm{p}=0.193)$

Conclusions: Gastrointestinal manifestations are very common in hATTR patients with Glu89Gln mutation and increase with disease duration. They are not associated with gender and onset of the disease and have no impact on patient survival. These results highlight the importance of a thorough evaluation of the GI function in patients with ATTR amyloidosis and should stimulate further studies on the phenotypic differences related to genotype and geographic origin.

Key words: amyloidosis - transthyretin - diarrhea - weight loss.

Abbreviations: ATTR: transthyretin amyloid; ATTRwt: transthyretin amyloid wild type; FAP: familial amyloidotic polyneuropathy; FC: fecal calprotectin; GI: gastrointestinal; hATTR - hereditary transthyretin amyloid; NYHA: New York Heart Association; THAOS: transthyretin amyloidosis outcomes survey; TTR: transthyretin.

\section{INTRODUCTION}

Transthyretin amyloid (ATTR) amyloidosis is an autosomal-dominant, adultonset, rare systemic disorder characterized by amyloid deposits formed by misfolded monomers of transthyretin (TTR). The disease can be hereditary (hATTR) or acquired (ATTRwt). The hereditary form of the disease is caused by point mutations in the TTR gene [1-2]. Diagnosis is challenging and may be delayed up to seven years from the initial appearance of symptoms [3].

Transthyretin, a 127 amino acids plasma transport protein that carries thyroxine and retinol-binding protein. It is produced primarily in the liver but also in the choroid plexus and retinal pigment epithelium, and is secreted into the blood, cerebrospinal fluid, and eye, respectively [4-6]. The native TTR is a homotetrameric complex, which can dissociate into monomers due to different factors

There are over 120 different TTR gene mutations identified worldwide, according to the online registry for mutations in hereditary amyloidosis [7]. The replacement of glutamate with 
glutamine at position 89 (ATTR Glu89Gln [p.Glu109Gln]) is the most commonly observed mutation in Bulgaria [8]. All of the Glu89Gln hATTR families in the country originate from an endemic region located in the South-West part of Bulgaria [8].

Transthyretin gene mutations destabilize TTR, leading to dissociation of tetramers and partial unfolding of resultant monomers. Variant TTR monomers aggregate to form amyloid fibrils $[4,9]$. Because of diffuse amyloid fibril deposition (e.g. in the extracellular space in peripheral nerves, heart, gastrointestinal tract, kidneys, eyes, meninges, vessels, and connective tissue of the transverse carpal ligament) [10], ATTR is associated with various symptoms, many of which are non-specific $[4,9]$.

Gastrointestinal (GI) manifestations are common in ATTR amyloidosis $[2,11]$ and are associated with diminished quality of life [2]. In some cases, GI symptoms are present even before the onset of the polyneuropathy and initial symptoms are often diarrhea, constipation, unintentional weight loss or nausea [2]. Moreover, different mutations are associated with varying patterns of clinical presentation, age of onset, and disease progression [12]. The disease course is highly variable and clinical manifestations are not necessarily uniform among carriers of the same TTR mutation and can vary even within the same family [13]. Gastrointestinal manifestations in patients with hATTR associated with Glu89Gln mutation have never been studied.

The aim of the present study was to explore the prevalence of GI manifestations in hATTR amyloidosis associated with Glu89Gln mutation.

\section{METHODS}

This is a prospective observational study. We recruited 78 patients with hATTR amyloidosis associated with Glu89Gln mutation from January 2012 to June 2018. The diagnosis of hATTR was defined by a documented TTR mutation through DNA analysis.

Symptoms were recorded as present or absent at the time of enrollment into the study. The duration of symptoms was calculated retrospectively from information provided by the patients at the time of enrollment. New York Heart Association (NYHA) classification was used to assess cardiac functional capacity. A comprehensive clinical neurological assessment (reflexes, sensation and muscle weakness) was performed and the stage of neurological disability was defined according to Familial Amyloidotic Polyneuropathy (FAP) scale [14]. Patients with ATTR amyloidosis and an age of $\geq 50$ years at symptom onset were defined as 'late-onset cases' in accordance to the current clinical praxis.

The GI symptoms checklist included the following items: early satiety, nausea, vomiting, constipation, alternating diarrhea/constipation, diarrhea, fecal incontinence and unintentional weight loss.

Throughout our diagnostic work-up in all the patients with GI manifestations upper and lower endoscopies with biopsies were performed. Amyloid deposits were identified in rectal biopsies by Congo red staining in combination with polarization microscopy. Fecal samples were collected within
1-2 days prior to the colonoscopy for fecal calprotectin (FC) level assessment in 14 patients (the test is not reimbursed by the National Healthcare Insurance Fund in Bulgaria).

\section{Statistical analysis}

The statistical analysis was performed using SPSS for Windows, Version 25.0. (SPSS Inc., Chicago, USA). Descriptive statistic for tabular and graphical presentation of results was used. Comparisons between cohorts and subgroups were carried out using the one-way ANOVA for the continuous variables. The chi-square test was used for categorical variables. Multiple regression analyses were carried out to identify potential predictors of GI symptoms. Kaplan-Meier curve for survival analysis was estimated. A p-value of $<0.05$ was considered statistically significant.

\section{Ethics}

The current study was approved by the Ethics Committee of “Tsaritsa Yoanna” University Hospital in Sofia, Bulgaria. Before initiating this study, written informed consent was obtained from all subjects. The study protocol conforms to the ethical guidelines of the 1975 Declaration of Helsinki (6th revision, 2008) as reflected in a priori approval by the Institution's Human Research Committee.

\section{RESULTS}

A total of 78 patients (mean age at onset $52.38 \pm 6.85,35$ 69; 40 males, 51.3\%) with hATTR amyloidosis associated with Glu89Gln mutation were enrolled in the study. All the patients had cardiac failure symptoms, 49 (62.8\%) were NYHA functional class 1 and $29(37.2 \%)$ - NYHA class 2. Neuropathy was observed in 76 (97.4\%) hATTR patients, 58 (74.4\%) of them were in neurological stage I, $15(19.2 \%)$ in stage II and 3 $(3.8 \%)$ in stage III. Carpal tunnel syndrome was described in 48 (61.5\%) patients. Fifty-four $(69.2 \%)$ were late-onset cases. The mean disease duration was $7.67 \pm 4.17$ years. The average delay in diagnosis was $5.17 \pm 4.5$ years. Characteristics of patients with hATTR amyloidosis associated with Glu89Gln mutation enrolled in the study can be seen in Table I.

The patients were followed every six months for an average period of 33 (4-72) months after diagnosis. Twentytwo patients (28\%) died, mainly because of advanced heart failure. The median age at death was 58.5 years (range: $52-72$ ) with a median disease duration of 90 months (range: 12-216). Gastrointestinal manifestations had no impact on survival $(\mathrm{p}=0.193)$ (Fig. 1).

Forty-two patients (53.8\%) reported at least one GI symptom. Diarrhea was the most frequently reported (30.8\%), followed by unintentional weight loss $(28.2 \%)$ and nausea (21.8\%). Fecal incontinence $(3.8 \%)$ was the least common symptom. The distribution of the individual GI symptoms in patients with hATTR associated with Glu89Gln mutation is presented in Table I.

Colonoscopy did not detect any mucosal changes; amyloid deposits were identified in rectal biopsies by Congo red staining in $15(35.7 \%)$ of the 42 patients with GI manifestations. Upper endoscopy evidenced retained food particles in the stomach in $12(28.6 \%)$ patients, suggesting abnormal gastric motor 
Table I. Characteristics of patients with hereditary transthyretin (hATTR) amyloidosis associated with Glu89Gln mutation enrolled in the study

\begin{tabular}{|c|c|c|}
\hline Characteristics & Absolute Number & Percentage (\%) \\
\hline Number of patients & 78 & 100 \\
\hline \multicolumn{3}{|l|}{ Sex } \\
\hline - Male & 40 & 51.3 \\
\hline - Female & 38 & 48.7 \\
\hline Age at onset, mean $\pm \mathrm{SD}$, range (years) & $52.38 \pm 6.85(35-69)$ & - \\
\hline - early-onset $<50$ years & 24 & 30.8 \\
\hline - late-onset $>50$ years & 54 & 69.2 \\
\hline Age at diagnosis, mean $\pm \mathrm{SD}$, range (years) & $56.88 \pm 6.65(36-73)$ & - \\
\hline Delay from first symptoms to diagnosis (years) & $5.17 \pm 4.5$ & - \\
\hline Disease duration & $7.67 \pm 4.17$ & - \\
\hline - disease duration $<5$ years & 19 & 24.4 \\
\hline - disease duration 5-10 years & 40 & 51.2 \\
\hline - disease duration $>10$ years & 19 & 24.2 \\
\hline Any GI symptom & 42 & 53.8 \\
\hline - Diarrhea & 24 & 30.8 \\
\hline - Unintentional weight loss & 22 & 28.2 \\
\hline - Nausea & 17 & 21.8 \\
\hline - Early satiety & 15 & 19.2 \\
\hline - Vomiting & 9 & 11.7 \\
\hline - Constipation & 7 & 9 \\
\hline - Diarrhea/constipation & 6 & 7.7 \\
\hline - Fecal incontinence & 3 & 3.8 \\
\hline Peripheral neuropathy & 76 & 97.4 \\
\hline - neurological stage I & 58 & 74.4 \\
\hline - neurological stage II & 15 & 19.2 \\
\hline - neurological stage III & 3 & 3.8 \\
\hline Cardiac failure symptoms & 78 & 100 \\
\hline - NYHA functional class I & 49 & 62.8 \\
\hline - NYHA functional class II & 29 & 37.2 \\
\hline Carpal tunnel syndrome & 48 & 61.5 \\
\hline Deceased patients & 22 & 28 \\
\hline
\end{tabular}

function. The mean level of FC in $14 \mathrm{hATTR}$ patients associated with Glu89Gln mutation was $187 \mu \mathrm{g} / \mathrm{g}$ (30-320).

No significant gender related difference in overall GI symptom prevalence was found (females $52.16 \%$, males $55 \%, \mathrm{p}=0.834)$. Numerical difference in symptom prevalence between men and women was found only for vomiting ( $5.3 \%$ vs. $17.9 \%, p=0.083)$, but this did not reach statistical significance. Moreover, no disease onset difference in GI symptom prevalence was found (early-onset $50 \%$, late-onset $55.6 \%, \mathrm{p}=0.650)$

After dividing the patients into groups with a disease duration of $<5$ years, 5-10 years and $>10$ years, respectively, the prevalence of GI symptoms was found to be significantly higher in later stages of the disease $(26.3 \%$ vs. $55.0 \%$ vs. $78.9 \%, \mathrm{p}=$ 0.005 ). This was due mainly regarding diarrhea (5.3\% vs. $40.0 \%$ vs. $36.8 \%, p=0.034)$ and alternating diarrhea/constipation $(0 \%$ vs. $55.0 \%$ vs. $78.9 \%, \mathrm{p}=0.021$ ), yet no significant differences were observed for the rest of GI symptoms.
Multiple regression analysis of factors associated with GI symptoms was performed and significant association was found only for duration of disease (OR 2.450, 95\% CI 1.084-5.538), whereas no significant association was found for male gender, early onset, NYHA status or neurological stage at diagnosis.

Nine patients $(11.5 \%)$ were eligible for treatment with tafamidis, an orphan drug used to delay loss of peripheral nerve function in adults with hATTR. The rest of the patients received symptomatic treatment. In the cases with upper GI symptoms we used low-dose erythromycin (50mg orally before meals) as first-line therapy, but we also prescribed metoclopramide 5-10mg before meals. We treated hATTR patients with constipation either with polyethylene glycol or with lactulose. Patients with diarrhea were advised to follow a fat-reduced diet and to take either loperamid or racecadotril or antibiotics. In cases with bile acid malabsorption, bile acid sequestrants such as cholestyramine ( $4 \mathrm{~g}$ before meals) could be effective. 


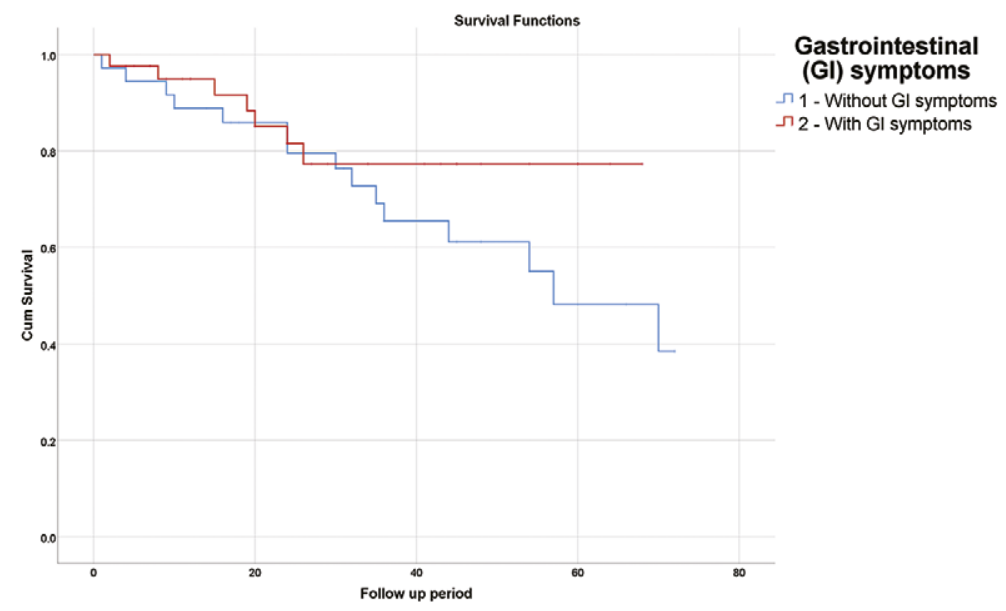

Fig. 1. Kaplan Meier survival analysis demonstrating that gastrointestinal manifestations have no impact on survival $(p=0.193)$ in ATTR amyloidosis patients with Glu89Gln mutation.

\section{DISCUSSION}

This is the first analysis describing the GI manifestations in patients with hATTR associated with Glu89Gln mutation. Glu89Gln is the most common mutation in the TTR gene in Bulgaria, where an endemic region in South-West part of the country is described [8]. Kirov et al. [8] observed a founder haplotype associated with Glu89Gln TTR mutation, which supported the hypothesis for the founder effect of the Glu89GIn mutation along with the presence of an endemic region for the mutation carriers in the country. This mutation is reported also in Sicily and Sardinia (Italy) [15] and in the European part of Turkey [12].

Despite the different geographic regions of origin, patients with Glu89Gln mutation show similar clinical manifestations: mixed phenotype with cardiac and neurological involvement with frequent autonomic disturbances and symptoms from the GI tract. In all described cohorts middle-aged population was affected. These findings differ from data regarding the most common mutation worldwide - Val30Met, in which a predominant neurological involvement with an early onset is typical for Portuguese patients and late onset with concomitant cardiac involvement is found in Swedish patients $[16,17]$.

In the current study we showed that GI symptoms were very common in hATTR patients with Glu89Gln mutation (53.8\%), much more frequent than their prevalence in the general population (i.e., 10-25\%) [18, 19]. The largest analysis of GI manifestations in ATTR patients was based on the Transthyretin Amyloidosis Outcomes Survey (THAOS) registry and showed that $59 \%$ of ATTR patients had at least one GI symptom [20]. There was no group of Glu89Gln patients in that study. However, the prevalence of GI symptoms in nonVal30Met was $56 \%$, which was similar to our results.

Diarrhea was the most frequently reported manifestation in our analysis. Typically, it was long-standing (more than 4 weeks), postprandial and sometimes was the presenting symptom of the disease, and this made the diagnosis challenging for the gastroenterologist. In some patients, undigested food fragments were observed in the stools. The mechanisms behind this diarrhea remain unexplained, but it is probably multifactorial. It is generally suggested that it arises due to motility disturbances of the GI tract caused by an autonomic neuropathy [21]. Other factors could be malabsorption of fat and bile acids as well as the frequent presence of bacterial overgrowth of the small bowel [22].

Unintentional weight loss was the second most common symptom. It could be observed even before the onset of GI or other symptoms [14]. The pathogenesis of weight loss is not fully elucidated, yet early satiety could be a contributing factor negatively affecting the patients' energy intake. Moreover, the amyloid deposition could also contribute to the weight loss through an increased metabolism, due to inflammatory reactions and oxidative stress $[23,24]$. Early satiety, as well as nausea and vomiting, are classic symptoms of gastric retention, which has also been demonstrated to be frequent in patients with ATTR amyloidosis [25].

It is well known that in the general population, functional GI disorders are more common in females [18, 19] and therefore, a female preponderance of GI symptoms was expected in hATTR patients. However, no significant gender related difference was observed for any GI symptom, in our study, which is consistent with a previous study [20].

In addition, there was no significant difference of GI symptoms' prevalence between early and late-onset cases with Glu89Gln mutation, which is inconsistent with previous findings in ATTR patients $[16,20]$. This means that GI manifestations were quite frequent irrespective of the disease onset in hATTR patients with the Glu89Gln mutation. On the other hand, GI symptoms (mainly diarrhea and alternating diarrhea/constipation) were more common with the duration of the disease, which probably reflects the development of a progressive autonomic neuropathy. Disease duration was the only significant predictor associated with GI symptoms in hATTR patients with Glu89Gln mutation. Furthermore, GI manifestations in hATTR patients with Glu89Gln mutation showed no effect on survival.

The reasons for the frequently occurring GI manifestations in hATTR patients with Glu89Gln mutation are not fully understood. However, malfunction of the autonomic and enteric nervous systems, including a depletion of the GI 
neuroendocrine cells and interstitial cells of Cajal, seems to be of importance $[21,25,26]$. In addition, it is generally suggested that GI symptoms arise due to motility disturbances of the GI tract caused by an autonomic neuropathy [22]. Moreover, small bacterial overgrowth and bile acid malabsorption contribute to the GI symptoms [27, 28]. The slightly elevated FC levels in a small group of our patients suggest an inflammatory component in the pathogenesis of GI complications in this disease. More studies are required to evaluate the role of FC in the diagnosis of ATTR amyloidosis with GI manifestations.

ATTR diagnosis is a real challenge for the gastroenterologist, due to the common GI disturbances and the broad differential diagnosis. The disease can easily be misdiagnosed with a number of other diseases of the GI tract and lead to diagnostic challenges in GI practice. Therefore, it is important for the gastroenterologists to include ATTR amyloidosis (with all known gene mutations for the geographical region they practice in) in their diagnostic work-up.

\section{CONCLUSIONS}

Gastrointestinal manifestations are very common in hATTR patients with Glu89Gln mutation and increase with disease duration. They are not associated with a specific gender or onset of the disease and have no impact on disease survival. These results highlight the importance of a thorough evaluation of the GI function in patients with ATTR amyloidosis and should stimulate further studies on the phenotypic differences related to genotype and geographic origin.

\section{Conflicts of interest: None to declare.}

Authors' contribution: R.N., S.S. and V.N. designed the study, enrolled and followed up the patients, performed statistical analyses, collected and analyzed the data and wrote the manuscript. M.G., T.T. A. K. and A.T. were responsible for patient diagnosis, analysis and treatment. I.T. supervised the whole team. All the authors approved the final version of the manuscript.

\section{REFERENCES}

1. Obici L, Kuks JB, Buades J, et al. Recommendations for presymptomatic genetic testing and management of individuals at risk for hereditary transthyretin amyloidosis. Curr Opin Neurol 2016;29:S27-S35 doi:10.1097/WCO.0000000000000290

2. Gertz MA. Hereditary ATTR amyloidosis: burden of illness and diagnostic challenges. Am J Manag Care 2017;23:S107-S112.

3. Adams D, Cauquil C, Labeyrie C. Familial amyloid polyneuropathy. Curr Opin Neurol 2017;30:481-489. doi:10.1097/WCO.0000000000000476

4. Ando Y, Coelho T, Berk JL, et al. Guideline of transthyretin-related hereditary amyloidosis for clinicians. Orphanet J Rare Dis 2013;8:31. doi:10.1186/1750-1172-8-31

5. Alemi M, Gaiteiro C, Ribeiro CA, et al. Transthyretin participates in betaamyloid transport from the brain to the liver-involvement of the lowdensity lipoprotein receptor-related protein 1? Sci Rep 2016;6:20164 doi:10.1038/srep20164

6. Sekijima Y, Ueda M, Koike H, Misawa S, Ishii T, Ando Y. Diagnosis and management of transthyretin familial amyloid polyneuropathy in
Japan: red-flag symptom clusters and treatment algorithm. Orphanet J Rare Dis 2018;13:6. doi:10.1186/s13023-017-0726-x

7. Rowczenio DM, Noor I, Gillmore JD, et al. Online registry for mutations in hereditary amyloidosis including nomenclature recommendations. Human Mutat 2014;35:E2403-E2412. doi:10.1002/humu.22619

8. Kirov A, Sarafov S, Pavlova Z, et al. Founder effect of the Glu89GIn TTR mutation in the Bulgarian population. Amyloid 2019;26:181-185. doi: 10.1080/13506129.2019.1634539

9. Hou X, Aguilar MI, Small DH. Transthyretin and familial amyloidotic polyneuropathy. Recent progress in understanding the molecular mechanism of neurodegeneration. FEBS J 2007;274:1637-1650. doi:10.1111/j.1742-4658.2007.05712.x

10. Takahashi K, Yi S, Kimura Y, Araki S. Familial amyloidotic polyneuropathy type 1 in Kumamoto, Japan: a clinicopathologic, histochemical, immunohistochemical, and ultrastructural study. Hum Pathol 1991;22:519-527. doi:10.1016/0046-8177(91)90227-g

11. Sekijima Y. Transthyretin (ATTR) amyloidosis: clinical spectrum, molecular pathogenesis and disease-modifying treatments. J Neurol Neurosurg Psychiatry 2015;86:1036-1043. doi:10.1136/jnnp-2014308724

12. Parman Y, Adams D, Obici L, et al. Sixty years of transthyretin familial amyloid polyneuropathy (TTR-FAP) in Europe: where are we now? A European network approach to defining the epidemiology and management patterns for TTR-FAP. Curr Opin Neurol 2016;29Suppl 1:S3-S13. doi:10.1097/WCO.0000000000000288

13. Conceição I, González-Duarte A, Obici L, et al. "Red-flag" symptom clusters in transthyretin familial amyloid polyneuropathy. J Peripher Nerv Syst 2016;21:5-9. doi:10.1111/jns.12153

14. Coutinho P, Martins da Silva A, Lopes Lima J, Resende Barbosa A. Forty years of experience with type I amyloid neuropathy. Review of 483 cases. In: Glenner G, Costa P, de Freitas A. (eds). Amyloid and Amyloidosis. Amsterdam: Excerpta Medica; 1980; 88-98.

15. Almeida MR, Ferlini A, Forabosco A, et al. Two transthyretin variants (TTR Ala-49 and TTR Gln-89) in two Sicilian kindreds with hereditary amyloidosis. Hum Mutat 1992;1: 211-215. doi:10.1002/ humu.1380010306

16. Conceição I, De Carvalho M. Clinical variability in type I familial amyloid polyneuropathy (Val30Met): comparison between lateand early-onset cases in Portugal. Muscle Nerve 2007;35:116-118. doi:10.1002/mus.20644

17. Suhr OB, Svendsen IH, Andersson R, Danielsson A, Holmgren G, Ranlov PJ. Hereditary transthyretin amyloidosis from a Scandinavian perspective. J Intern Med 2003;254:225-235. doi:10.1046/j.1365-2796.2003.01173.x

18. Boyce PM, Talley NJ, Burke C, Koloski NA. Epidemiology of the functional gastrointestinal disorders diagnosed according to Rome II criteria: an Australian population-based study. Intern Med J 2006;36:2836. doi:10.1111/j.1445-5994.2006.01006.X

19. Dong YY, Chen FX, Yu YB, et al. A school-based study with Rome III criteria on the prevalence of functional gastrointestinal disorders in Chinese college and university students. PLoS ONE 2013;8:e54183. doi:10.1371/journal.pone.0054183

20. Wixner J, Mundayat R, Karayal ON, Anan I, Karling P, Suhr OB. THAOS: gastrointestinal manifestations of transthyretin amyloidosis common complications of a rare disease. Orphanet J Rare Dis 2014;9:61. doi:10.1186/1750-1172-9-61

21. Ikeda S, Yanagisawa N, Hongo M, Ito N. Vagus nerve and celiac ganglion lesions in generalized amyloidosis. A correlative study of familial amyloid polyneuropathy and AL-amyloidosis. J Neurol Sci 1987;79:129-139. Doi:10.1016/0022-510x(87)90267-x 
22. Wixner J, Suhr OB, Anan I. Management of gastrointestinal complications in hereditary transthyretin amyloidosis: a single-center experience over 40 years. Expert Rev Gastroenterol Hepatol 2018;12:73 81. doi:10.1080/17474124.2018.1397511

23. Sousa MM, Cardoso I, Fernandes R, Guimaraes A, Saraiva MJ. Deposition of Transthyretin in Early Stages of Familial Amyloidotic Polyneuropathy. Am J Pathol 2001;159:1993-2000. doi:10.1016/s00029440(10)63050-7

24. Saraiva MJ. Cellular consequences of transthyretin deposition. Amyloid 2003;10 Suppl 1:13-16.

25. Wixner J, Karling P, Rydh A, et al. Gastric emptying in hereditary transthyretin amyloidosis: the impact of autonomic neuropathy.
Neurogastroenterol Motil 2012;24:1111-e568. doi:10.1111/j.13652982.2012.01991.X

26. Wixner J, Obayashi K, Ando Y, Karling P, Anan I. Loss of gastric interstitial cells of Cajal in patients with hereditary transthyretin amyloidosis. Amyloid 2013;20:99-106. doi:10.3109/13506129.2013.787985

27. Steen L, Ek B. Familial amyloidosis with polyneuropathy. A long-term follow-up of 21 patients with special reference to gastrointestinal symptoms. Acta Med Scand 1983;214:387-397.

28. Suhr O, Danielsson A, Steen L. Bile acid malabsorption caused by gastrointestinal motility dysfunction? An investigation of gastrointestinal disturbances in familial amyloidosis with polyneuropathy. Scand J Gastroenterol 1992;27:201-207. doi:10.3109/00365529208999949 